\title{
Philosophiques
}

\section{Les arguments transcendantaux et le problème de la justification de la normativité morale}

\section{Stélios Virvidakis}

Volume 28, numéro 1, printemps 2001

La nature des normes

URI : https://id.erudit.org/iderudit/004996ar

DOI : https://doi.org/10.7202/004996ar

Aller au sommaire du numéro

\section{Éditeur(s)}

Société de philosophie du Québec

ISSN

0316-2923 (imprimé)

1492-1391 (numérique)

Découvrir la revue

Citer cet article

Virvidakis, S. (2001). Les arguments transcendantaux et le problème de la justification de la normativité morale. Philosophiques, 28(1), 109-128.

https://doi.org/10.7202/004996ar
Résumé de l'article

La théorie de la connaissance des années 60 et 70 a été marquée par l'emploi des arguments transcendantaux de toutes sortes. Ces arguments pourraient servir à défendre l'applicabilité de nos concepts fondamentaux qui font l'objet d'une variété d'attaques sceptiques. Malheureusement, la prise de conscience des difficultés mettant en cause l'efficacité ou même la validité des arguments transcendantaux a progressivement conduit à leur abandon, quoique certains philosophes n'aient jamais cessé de les utiliser. Mon analyse s'inspire du renouveau récent de l'intérêt pour l'argumentation transcendantale dans le domaine de la raison pratique, et en particulier de l'élaboration d'un modèle de ce genre d'argumentation par $\mathrm{A}$. W. Moore. Je procède à l'examen d'un argument de forme transcendantale développé par Christine Korsgaard afin de justifier l'autorité des normes morales. Je tente d'évaluer l'efficacité de cet argument et du modèle général auquel il se conforme, de le comparer à des approches alternatives et d'étudier les rapports de ses prémisses et de sa conclusion avec des prises de position réalistes et anti-réalistes en métaéthique. 


\title{
Les arguments transcendantaux et le problème de la justification de la normativité morale
}

\author{
STÉLIOS VIRVIDAKIS \\ Université d'Athènes \\ svirvid@cc.uoa.gr
}

\begin{abstract}
RÉSUMÉ. - La théorie de la connaissance des années 60 et 70 a été marquée par l'emploi des arguments transcendantaux de toutes sortes. Ces arguments pourraient servir à défendre l'applicabilité de nos concepts fondamentaux qui font l'objet d'une variété d'attaques sceptiques. Malheureusement, la prise de conscience des difficultés mettant en cause l'efficacité ou même la validité des arguments transcendantaux a progressivement conduit à leur abandon, quoique certains philosophes n'aient jamais cessé de les utiliser. Mon analyse s'inspire du renouveau récent de l'intérêt pour l'argumentation transcendantale dans le domaine de la raison pratique, et en particulier de l'élaboration d'un modèle de ce genre d'argumentation par A. W. Moore. Je procède à l'examen d'un argument de forme transcendantale développé par Christine Korsgaard afin de justifier l'autorité des normes morales. Je tente d'évaluer l'efficacité de cet argument et du modèle général auquel il se conforme, de le comparer à des approches alternatives et d'étudier les rapports de ses prémisses et de sa conclusion avec des prises de position réalistes et anti-réalistes en métaéthique.
\end{abstract}

\begin{abstract}
There is a revival of interest in transcendental arguments, which used to be very popular in recent decades but were progressively abandoned because of doubts concerning their plausibility and even their validity, although of course some philosophers never ceased to use them. My paper deals with a particular model of transcendental arguments which can be employed in the area of practical philosophy, elaborated by A.W. Moore. I examine an application of this model in the construction of a complex argument by Christine Korsgaard which aims at establishing the authority of moral norms. I discuss its structure and the relations of its premises and of its conclusion to certain realist and antirealist positions in metaethics, I compare it to alternative approaches to the same problem, and I try to evaluate its strengths and weaknesses.
\end{abstract}

\section{Introduction}

La théorie de la connaissance analytique des années 60 et 70 a été marquée par l'étude et l'emploi des arguments transcendantaux. Suivant la lecture de Kant et l'approche de la métaphysique descriptive élaborées par Peter Strawson, on considérait comme transcendantal tout argument visant à mettre en lumière des conditions de possibilité de l'expérience. Des arguments de ce genre pourraient éventuellement permettre la réfutation de plusieurs formes de scepticisme et aboutir à des conclusions portant sur les éléments de base d'une métaphysique de l'expérience. Malheureusement, la prise de conscience d'une série de problèmes concernant leur structure et surtout l'inter- 
prétation de leurs prémisses principales a conduit à remettre en cause leur plausibilité, ou même leur validité, et à abandonner la plupart des projets épistémologiques plus ou moins ambitieux pour le développement d'une méthode transcendantale. Cependant, on n'a pas cessé de construire des arguments cherchant à établir les conditions de possibilité de la compréhension de divers aspects de notre expérience et de l'usage de nos concepts fondamentaux dans le domaine de l'ontologie, de la philosophie de l'esprit et de la philosophie du langage. En effet, on témoigne aujourd'hui d'un renouveau du débat sur les perspectives et les limites de l'argumentation transcendantale ${ }^{1}$. Ce renouveau intéresse aussi les philosophes qui participent aux discussions métaéthiques, dans la mesure où l'argumentation en question pourrait servir à élucider et à défendre certaines conceptions de la raison pratique.

Dans ce qui suit, mon analyse a précisément pour objet l'élaboration et l'emploi d'un modèle transcendantal pour l'argumentation en métaéthique qui se prête à la justification de l'autorité des normes morales. Il s'agit du modèle de ce que A. W. Moore appelle « des arguments transcendantaux conatifs ${ }^{2}$ » et de son application à la reconstruction et à l'évaluation de certains arguments des philosophes qui aspirent à défendre des formes d'objectivisme plus ou moins fort en éthique. La mise en évidence des impasses du débat sur le réalisme moral rend nécessaire l'examen critique de toute stratégie argumentative qui pourrait faciliter la compréhension de la problématique actuelle et contribuer à la résolution des controverses métaéthiques 3 . Je veux donc me concentrer sur un exemple d'argumentation transcendantale dans le domaine de la philosophie pratique afin d'en examiner la structure et les prémisses principales et de prendre en considération ses rapports avec certaines positions réalistes et antiréalistes portant sur les questions de l'origine, de l'objectivité et de l'autorité des normes morales.

\section{Les arguments transcendantaux conatifs}

Selon Moore, on pourrait résumer les caractéristiques principales des arguments transcendantaux par l'esquisse de la forme suivante $(\mathrm{T})$ :

1. Pour une présentation récente des discussions concernant les arguments transcendantaux, voir Stern, Robert, dir., Transcendental Arguments : Problems and Prospects, Oxford, Clarendon Press, 1999. Ce recueil des communications à un colloque de 1997 sur les arguments transcendantaux contient aussi une bibliographie détaillée qui inclut dans sa liste les premiers ouvrages sur ce sujet.

2. Moore, A. W., "Conative Transcendental Arguments and the Question Whether There Can Be External Reasons ", dans Stern, dir., Transcendental Arguments : Problems and Prospects, p. 271-292.

3. Pour une discussion critique du débat sur le réalisme moral, voir Virvidakis, Stélios, La robustesse du bien, Essai sur le réalisme moral, Nîmes, Éditions Jacqueline Chambon, 1996. Cf. aussi Ogien, Ruwen, dir., Le réalisme moral, Paris, Presses Universitaires de France, 1999. 
(1t) $p$

(2t) Il ne serait pas possible que $p$ si nous ne pensions pas que $q$

(3t) Nous devons ${ }^{4}$ penser que $q$

(4t) Il est vrai que $q^{5}$.

En général, « $p$ » se réfère à une façon de se représenter la réalité, que l'on pourrait décrire comme une sorte d'expérience consciente ou intelligible, telle que la conscience de soi, l'emploi des concepts, la formation des croyances et la formulation des assertions ou la communication par le langage, et « $q$ » désigne un aspect de la réalité faisant souvent l'objet de quelque doute sceptique. Il y a bien sûr plusieurs problèmes, auxquels on a déjà fait allusion, qui portent sur la vérité de (2t), sur la modalité invoquée dans (2t) et (3t) et la nécessité attribuée à $q$, et surtout sur la validité de la transition de (3t) à (4t), effectuée sans le soutien d'une prémisse vérificationniste ou idéaliste ${ }^{6}$.

La variante de cette structure du raisonnement transcendantal qui constitue le modèle des arguments " conatifs » se base sur l'hypothèse de la substitution d'un état mental du genre du désir à celui du genre de la croyance. C'est pourquoi Moore propose l'emploi du terme générique "désirer » (que q), qui pourrait signifier " espérer, vouloir, souhaiter ", ou même " considérer comme juste et approprié ». Ici, il convient de noter que, selon la suggestion de Moore, cette modification de l'approche des états mentaux en question n'entraînerait pas forcément le besoin de transformer toutes les prémisses des arguments transcendantaux afin de les adapter à la nature conative des composantes de la réflexion pratique. Le contenu de $p$ et de $q$ pourrait correspondre à des états mentaux qu'on serait autorisé à concevoir comme des attitudes de croyance et de désir à la fois, comme par exemple "être un agent rationnel ». Ce qui importe c'est de remplacer la dérivation des conditions de possibilité qui concernent ce que l'on doit penser ou croire par des conditions de possibilité qui indiquent ce que l'on doit désirer, dans un sens plutôt large du terme ${ }^{7}$. Aussi pourrait-on adopter le modèle suivant $(\mathrm{C})$ :

4. Dans les formulations des prémisses des arguments qui suivent, j’emploie le verbe " devoir » pour traduire l'anglais « must », quoique le terme n'ait aucune implication déontique ou morale ni dans le schéma général, ni dans le cas du modèle conatif, et exprime une nécessité transcendantale plus forte que seule exprimée par le sens de " ought ». Je veux souligner la référence au sujet ( "nous »), et j'ai choisi le mot " devoir » au lieu de " être obligé à » ou même de « il faut que " (malgré ses connotations de neutralité et de force modale) pour des raisons stylistiques. Nous reviendrons plus bas aux nuances de la modalité en question.

5. Moore, "Conative Transcendental Arguments... ", p. 271. Le choix du pronom personnel «nous » au lieu de « on » (indéfini) — « we » au lieu de « one »— facilite la mise en évidence du problème de l'extension et de la référence exacte du terme employé pour désigner le sujet des prémisses et des conclusions des arguments transcendantaux.

6. En ce qui concerne ces critiques, voir Stroud, Barry, "Transcendental Arguments ", The Journal of Philosophy, 65, 1968, p. 241-256, et Virvidakis, Stélios, Transcendental Arguments, Transcendental Idealism and Scepticism, (thèse de doctorat inédite), Princeton, 1984.

7. Moore, «Conative Transcendental Arguments... », p. 272-273. Moore parle de l'opposition entre représentation et " conation ». Il serait peut-être plus juste de renvoyer au contraste 
(1c) $p$

(2c) Il ne serait pas possible que $p$ si nous ne désirions pas que $q$

(3c) Nous devons désirer que $q$

(4c) Il est désirable que $q^{8}$.

Comme le constate Moore, les arguments conatifs présentent en même temps des avantages et des défauts particuliers comparativement aux autres arguments transcendantaux. D'une part, la transition de $(3 c)$ à $(4 c)$ peut s'effectuer sans l'appel à une prémisse vérificationniste ou idéaliste qui nous permettrait d'assurer la conformité du contenu ou de la structure de nos états mentaux, dont on aurait démontré le caractère nécessaire, à une réalité tout à fait indépendante. En éthique, il est très difficile de soutenir des positions réalistes fortes, stipulant la possibilité d'une indépendance ou divergence radicale des faits moraux dont il est question par rapport à nos capacités épistémiques de les atteindre. Il est probable que les partisans de toute une variété de conceptions réalistes et cognitivistes modérées s'accorderaient avec les défenseurs de certaines alternatives antiréalistes pour reconnaître la légitimité de la dérivation de $(4 \mathrm{c})$ à partir de $(3 \mathrm{c})^{9}$. D’autre part, la nécessité de la conclusion $(4 \mathrm{c})$ que l'on tâche d'établir est apparemment beaucoup plus faible que la nécessité de la conclusion (4t) de la plupart des arguments transcendantaux en théorie de la connaissance et en métaphysique. En tout cas, il s'agit d'une sorte de nécessité conditionnelle qui dépend du statut de la première prémisse $p$, aussi bien que de la relation spécifiée respectivement par (2t) et (2c). Sans prendre en considération les difficultés concernant la nature de la modalité attribuée aux

traditionnel entre croyances et désirs, conçus comme deux sortes d'attitudes propositionnelles. On aurait alors affaire à deux espèces de représentation qui diffèrent en ce qui concerne ce que l'on pourrait appeler leur "direction d'ajustement ». Dans le cas des croyances, «l'esprit s'ajuste au monde », puisque les croyances vraies doivent se conformer à la réalité telle qu'elle est, tandis que les désirs indiquent comment " le monde devrait s'ajuster à notre esprit ", c'està-dire se transformer afin de se conformer à notre volonté (cf. Platts, Mark, Ways of Meaning : An Introduction to a Philosophy of Language, London, Routledge and Kegan Paul, 1979, p.265-7 ; Anscombe, Elizabeth, Intention, Oxford, Blackwell, 1957, \$32). Moore insiste sur la composante conative de sa conception générale du " désir ", tout en reconnaissant le besoin de prendre en considération l'aspect représentationnel qu'il est prêt à relier à nos tendances réalistes. Cf. Moore, "Conative Transcendental Arguments... », p. 277, n. 16.

8. Ibid., p.274. Moore remarque le parallèle apparent entre l'inférence de (3c) à (4c) et l'argument supposément fallacieux de John Stuart Mill, soutenant que le fait que les gens désirent quelque chose prouve que cette chose est (objectivement) désirable, tout comme le fait que les gens voient quelque chose prouve que cette chose est visible. En effet, cet argument avait été analysé par G.E. Moore comme un cas caractéristique de sophisme naturaliste. (Mill, John Stuart, Utilitarianism, London, Fontana, 1962, p. 288-289, Moore, G. E., Principia Ethica, Cambridge, Cambridge University Press, 1959, p. 67). Cependant, (3c) indique déjà ce que nous devons désirer, quoique, comme on le verra plus bas, la nature de la nécessité impliquée par nos prémisses ne soit pas tout à fait claire.

9. Pour un premier compte rendu de certaines formes de réalisme moral modéré, voir Virvidakis, Stélios, "Stratégies de modération du réalisme moral », dans Ogien, Ruwen, dir. Le réalisme moral, Paris, Presses Universitaires de France, 1999, p. 420-456. Cf. aussi, Virvidakis, La robustesse du bien, p. 262-276. 
prémisses, aussi bien qu'à la conclusion que l'on prétend en dégager, qui se présente comme une sorte d'hybride transcendantal comportant des aspects conceptuels et psychologiques à la fois, on remarque que dans le cas des arguments conatifs, on ne parvient pas à assurer la vérité, et $a$ fortiori la vérité nécessaire — dans un sens transcendantal — de $p$. En plus, il n'est pas du tout évident que " désirer » que $q$ constitue une condition nécessaire de $p$.

D'un point de vue dialectique, même le sceptique le plus obstiné ne pourrait pas facilement nier le fait qu'il est, par exemple, conscient de son expérience subjective ou qu'il est capable d'exprimer ses positions de façon intelligible. Par contre, le rejet de toute assertion qui stipule, par exemple, que nous sommes des agents rationnels, ou que nous sommes contraints à vouloir assumer cette rationalité - la "désirer » — ou lui attribuer une valeur fondamentale n'entraîne pas une contradiction dialectique ou pragmatique quelconque qui pourrait servir de prémisse pour une reductio du scepticisme en éthique. La clause initiale de notre raisonnement n'engage pas un amoraliste ou un nihiliste déterminé, qui se sentirait capable de poursuivre le débat sans partager ce que nous considérons comme des présupposés communs.

Néanmoins, d'après Moore, on ne devrait pas hésiter à avoir recours aux arguments transcendantaux conatifs afin d'essayer de défendre certaines conceptions des éléments de base et du fonctionnement de la raison pratique, pourvu qu'on les traite comme des tentatives de justification plus ou moins souples plutôt que comme des démonstrations strictes et définitives. Il s'agirait d'une "déduction » transcendantale d'inspiration kantienne, mais dans le sens assez large d'un processus de légitimation de l'emploi d'un concept ou d'un principe auquel on reconnaîtrait un rôle central dans notre pensée éthique ${ }^{10}$. Plus précisément, le modèle proposé par Moore fournirait une telle déduction du caractère " désirable " de l'adoption d'un concept ou d'un principe de ce genre. En effet, suivant son analyse, l'application du modèle transcendantal conatif pourrait avant tout permettre de surmonter l'opposition entre l'internalisme et l'externalisme concernant l'existence des raisons d'agir indépendantes ou externes à l'ensemble des motivations subjectives qui dépendent des désirs subjectifs — d'un agent ${ }^{11}$. Selon Moore, la contro-

10. Moore, "Conative Transcendental Arguments... », p. 282 et 289. En ce qui concerne le sens exact du terme Deduktion chez Kant, voir Henrich, Dieter, "Kant's Notion of a Deduction and the Methodological Background of the First Critique », dans Förster, Eckhart, dir., Kant's Transcendental Deductions, Stanford, Stanford University Press, 1989. Henrich trace les origines juridiques de la notion d'une « déduction » visant à établir la légitimité des prétentions à un droit. Pour une interprétation des arguments transcendantaux dans la philosophie pratique de Kant, voir, Benton, Robert, J., Kant's Second Critique and the Problem of Transcendental Arguments, The Hague, Martinus Nijhoff, 1977.

11. Il ne faut pas confondre la distinction entre l'externalisme et l'internalisme dans ce sens (voir Williams, Bernard, "Internal and External Reasons ", dans Moral Luck, Cambridge, Cambridge University Press, 1981, p. 101-113 et McDowell, John, « Might There Be External Reasons ? » dans Altham, J.E.J. et Harrison, Ross, dir., World, Mind and Ethics : 


\section{$114 \cdot$ Philosophiques / Printemps 2001}

verse perd sa pertinence et même son contenu s'il y a des choses que nous ne pouvons pas éviter de désirer en tout cas, étant donné notre nature rationnelle. À part cette tentative de résoudre le problème de l'existence des raisons externes, Moore procède à l'énumération d'une variété d'arguments qui semblent se conformer à son modèle, sans toutefois tâcher de les reconstruire et de se prononcer sur leurs mérites et leurs faiblesses particulières. Il est prêt à anticiper plusieurs difficultés auxquelles on doit faire face si on décide de développer ces arguments, mais il se contente de discuter certaines questions plutôt générales concernant l'extension du sujet pluriel des prémisses ( «nous ») et la référence collective ou distributive aux états mentaux de tous les individus impliqués ${ }^{12}$.

Il est clair que cette présentation sommaire des suggestions de Moore ne parvient pas à rendre justice à la subtilité de son raisonnement. Cependant, on n'a pas besoin d'une analyse détaillée pour observer que le modèle proposé soulève déjà des problèmes portant sur l'applicabilité et l'efficacité des procédures argumentatives transcendantales dans le domaine de la philosophie morale. En effet, on ne pourrait pas tenter de les résoudre sans prendre en considération des tentatives spécifiques d'employer des arguments de ce genre afin d'en élucider les caractéristiques et d'en évaluer la plausibilité et la force.

Dans les sections suivantes, j'examinerai une approche de la justification de l'autorité des normes morales qui pourrait être considérée comme transcendantale et qui dérive de la pensée de Kant. Ce qui m’intéresse, ce n'est pas tellement la fidélité de l'interprétation des notions kantiennes et des arguments qui les étayent, mais plutôt leur fonction dans le contexte des débats contemporains. C'est pourquoi j'éviterai presque totalement les questions exégétiques, en me limitant à la discussion critique des reconstructions actuelles. En particulier, je propose d'étudier l'argumentation de Christine Korsgaard portant sur les sources de la normativité, qualifiée de transcendantale par elle-même de façon explicite et mentionnée par Moore parmi les exemples de réalisation de son schéma. J'aimerais isoler la structure et les prémisses de base de son raisonnement afin d'essayer d'aborder les problèmes que nous avons identifiés. Je veux aussi me référer brièvement à des conceptions alternatives de la raison pratique et des normes morales. Ces

Essays on the Ethical Philosophy of Bernard Williams, Cambridge, Cambridge University Press, p. 68-85) avec l'opposition entre les notions de l'internalisme et de l'externalisme signifiant respectivement l'affirmation de la présence ou de l'absence d'un lien interne et nécessaire entre les jugements moraux et la volonté ou l'action. Sur les variantes de cette opposition et son importance pour le débat sur le réalisme moral, voir Virvidakis, La robustesse du bien, p. 128-133, 191-195, 251-256, et Smith, Michael, The Moral Problem, Oxford, Blackwell, 1994. Voir aussi Dancy, Jonathan, Moral Reasons, Oxford, Blackwell, 1993, app. I et Nagel, Thomas, "L'éthique et la motivation humaine ", dans Ogien, Ruwen, dir., Le réalisme moral, p. 362-381 (extrait de Nagel, Thomas, The Possibility of Altruism, Princeton, Princeton University Press, 1970.).

12. Moore, "Conative Transcendental Arguments... » p.282-292. Voir ci-dessus notes 4 et 5. 
conceptions, quoique également d'inspiration rationaliste et jusqu'à un certain point tributaires de la pensée kantienne, sont soutenues par des arguments qui, au moins suivant une première lecture, ne semblent pas relever du modèle transcendantal ou être réductibles à sa structure. En les mettant en comparaison avec les idées centrales et la démarche argumentative de Korsgaard, on pourrait aspirer à une meilleure compréhension et appréciation de la spécificité de la stratégie transcendantale en métaéthique.

\section{Une tentative de déduction transcendantale de la normativité morale}

\subsection{La «question normative »}

$\mathrm{La}$ " question normative » à laquelle nous devons répondre est, selon Korsgaard, la question centrale qui concerne la justification de nos obligations morales :

Quand nous sommes en quête d'une fondation philosophique de la moralité, nous ne cherchons pas seulement une explication de nos pratiques morales. Nous voulons apprendre ce qui justifie les demandes qui nous sont adressées par la morale ${ }^{13}$.

Il s’agit du problème qui préoccupe la pensée des philosophes depuis l'époque de Socrate et de Platon : pourquoi accepter les demandes de la morale ? Autrement dit, pourquoi adopter un point de vue imposant la régulation de notre comportement selon des exigences qui, souvent, vont à l'encontre de nos désirs et de nos inclinations égoïstes ? Bref, pourquoi être moral ? Ayant perdu l'appui des conceptions métaphysiques de la philosophie grecque qui nous permettaient de croire que les valeurs existent dans le monde, nous ne pouvons plus aspirer à l'acquisition de la vertu éthique, conçue comme la réalisation d'une excellence plus ou moins naturelle. Nous sommes réduits à une morale du devoir ou de l'obligation qui constitue l'héritage de la pensée judéo-chrétienne ${ }^{14}$. Il devient très difficile de répondre

13. Cf. Korsgaard, Christine, avec G. A. Cohen. [et al.], The Sources of Normativity, Cambridge, Cambridge University Press, $1996, \mathbb{\$} 1.2 .1$ p. 9-10. Pour son interprétation des textes de la philosophie morale de Kant, voir les articles dans Korsgaard, Christine, Creating the Kingdom of Ends, Cambridge, Cambridge University Press, 1996. Voir aussi les discussions sur l'approche interprétative de Korsgaard dans le Symposium consacré à Creating the Kingdom of Ends, Ethics 109, 1998, p. 5- 66, qui sont très utiles pour la meilleure compréhension de son ouvrage The Sources of Normativity.

14. Pour une analyse de ce processus historique et de ses conséquences -désastreuses selon un certain nombre de philosophes prêchant le retour à une éthique de la vertu, si bien-sûr un tel retour s'avérait possible, voir les écrits de Elizabeth Anscombe, de Iris Murdoch, et de Alasdair MacIntyre. Cf. Virvidakis, La robustesse du bien, p. 38-49. En ce qui concerne le problème de l'emploi des termes "morale » et "éthique ", que l'on propose parfois de distinguer afin de désigner respectivement une notion plus étroite portant sur le code de l'obligation (l'ordre du « juste ») et une notion plus large qui relève de la vie éthique (l'ordre du 
à la question normative dès lors que l'on est témoin de la sécularisation progressive des sociétés modernes, qui entraîne l'éclipse de la métaphysique chrétienne et l'effondrement général de la foi religieuse.

Suivant le compte-rendu historique de Korsgaard, on pourrait isoler quatre réponses principales à la question normative, réponses formulées et élaborées par les philosophes de l'époque moderne jusqu'à nos jours : a) le volontarisme attribué à Pufendorf et à Hobbes selon lequel la normativité dérive d'une volonté législative qui ressemble à l'autorité divine et s'exprime sur le plan politique ; b) le réalisme qui se base sur l'idée de l'existence d'une dimension d'entités intrinsèquement normatives qui fournissent des raisons d'agir appréhendées par une sorte d'intuition rationnelle et décrites par nos jugements moraux vrais. Cette conception est défendue par les intuitionnistes en éthique, de Clarke jusqu'à Moore, Prichard et Ross, et est représentée aujourd'hui par Nagel; c) l'" endossement réfléchi » des raisons d'agir qui sont censées être conformes à notre nature, pratiqué par une variété de philosophes tels que Hutcheson, Hume et John Stuart Mill, et adapté à ses fins et développé de façon consciente par Kant ; d) l'appel à l'autonomie proposé par Kant, accepté aussi par des philosophes, tel que Rawls, qui se réclament de toute forme de constructivisme d'origine kantienne, et élaboré par Korsgaard elle-même comme la solution la plus appropriée à la conception du monde et à la sensibilité des temps modernes.

Ici, nous ne pouvons pas examiner la plausibilité de la classification adoptée par Korsgaard et la façon dont elle interprète les diverses positions des penseurs modernes qu'elle étudie. Nous sommes surtout intéressés par sa propre interprétation de la réponse kantienne et plus particulièrement par sa version de la déduction transcendantale de la normativité morale, qui constitue un exemple caractéristique d'argument transcendantal conatif.

\footnotetext{
«bien »), voir Ricoeur, Paul, «Éthique et morale », Pouvoirs, no 65, 1993, p. 5-18. Comme le remarque Charles Larmore il faudrait peut-être reconnaître la " priorité du bien par rapport au juste » seulement dans le contexte de l'éthique ancienne de la vertu, sans se référer au contraste entre théories conséquentialistes (comme l'utilitarisme) et théories déontologiques (comme la théorie kantienne) qui se devéloppent dans l'ère moderne. (Larmore, Charles, Modernité et morale, Paris, Presses Universitaires de France, 1993, p.43-69). Voir aussi Virvidakis, La robustesse du bien, p.7n, 209n. Cependant, malgré le fait que Korsgaard se concentre sur le problème de l'obligation et son approche, aussi bien que les autres positions que nous allons prendre en considération, se limitent à la discussion des normes de la raison pratique et des valeurs qui fondent ces normes, de façon qui semble renvoyer au sens plus étroit d'un code moral, je veux employer les termes "morale » et "éthique ", comme des variantes stylistiques sans signification particulière, sauf au cas où les auteurs adoptent une distinction d'usage de manière explicite. Sur les relations de priorité logique, sémantique, épistémologique ou métaphysique entre les valeurs et les normes, voir Ogien, Ruwen, "Normes et Valeurs ", dans Canto-Sperber, Monique, dir., Dictionnaire d'éthique et de philosophie morale, Paris, Presses Universitaires de France, 1996, p. 1056-1064.
} 


\subsubsection{L'argument central de Korsgaard}

Les prémisses de base de l'argumentation de Korsgaard, suivant une première lecture (A) que l'on peut ajuster au schéma de Moore se réfèrent au fait que nous agissons en reconnaissant la validité et la force des raisons déterminées par nos facultés intellectuelles $(p)$, aussi bien qu'aux conditions de possibilité de cet agir rationnel, et en particulier le « désir » qui a comme objet l'existence de notre " humanité » $(q)^{15}$ :

1a) Nous agissons de façon rationnelle.

2a) Nous ne pourrions pas agir de façon rationnelle, si nous ne désirions pas l'existence de ce que nous reconnaissons comme notre humanité.

3a) Nous devons désirer l'existence de notre humanité.

4a) Il est désirable que notre humanité existe.

La notion du " désir ", dans le sens large d'état conatif, renvoie dans ce cas à l'appréciation, ou plus précisément à l'affirmation, ou l'attribution de la valeur ("valuing ") ${ }^{16}$. La conception de notre humanité se réfère à notre qualité humaine essentielle et commune à tous. L'argument (B) pourrait alors servir à établir la relation constitutive entre l'agir rationnel et l'attribution de la valeur à notre humanité :

1b) Nous agissons de façon rationnelle.

2b) Nous ne pourrions pas agir de façon rationnelle si nous n'attribuions pas de valeur à notre humanité.

3b) Nous devons attribuer de la valeur à notre humanité.

4b) Notre humanité a de la valeur.

Avant de procéder à l'analyse des difficultés soulevées par ce raisonnement plutôt dense et par sa formulation, il importe de noter que l'argument se développe par l'intermédiaire des prémisses supplémentaires qui relient l'agir rationnel avec la valeur attribuée à notre humanité. Ces prémisses se réfèrent à nos capacités de réflexion et au concept de notre "identité normative » ou, plus précisément, du concept du caractère normatif de notre

15. Korsgaard elle-même caractérise son argument comme transcendantal. Cf. The Sources of Normativity, p. 123-125sq. La reconstruction qui suit est une variante d'après le modèle de Moore. Cf. «Conative Transcendental Arguments... ", p. 291.

16. Ici, nous voulons adopter une conception de la valeur intrinsèque et noninstrumentale, quoique la valeur en question soit aussi de nature relationnelle, dans la mesure où elle est supposée dépendre de l'activité de la volonté humaine. Nous avons affaire à une approche anti-réaliste, projectiviste ou constructiviste, et en même temps à une perspective épistémologique plus ou moins fondationnaliste. La valeur attribuée à notre humanité devrait être inconditionnelle et absolue, puisqu'elle serait la condition de toute autre évaluation dans le monde des faits, bien qu'elle dérive d'une source purement subjective ou plutôt anthropocentrique. En plus, il faut éviter la confusion éventuelle entre la notion générique du "désir " au sens large de tout état mental conatif (qui pourrait bien sûr impliquer une composante épistémique, et plus précisément doxastique), proposé par Moore, et le concept du désir, au sens étroit d'un état affectif qui relève entièrement de l'ordre de l'appétition, suivant la tradition de la psychologie humienne. 
identité pratique en tant qu'agents rationnels. On pourrait donc adopter une reconstruction beaucoup plus détaillée $(\mathrm{V})$ :

1v) Nous agissons de façon rationnelle.

$2 v)$ Nous ne pourrions pas agir de façon rationnelle si nous n'étions pas capables de soumettre nos inclinations à une épreuve critique afin de savoir si et quand elles constituent des raisons d'agir fournissant des motifs pour nos actions particulières.

3v) Nous ne pourrions pas soumettre nos inclinations à une épreuve critique si nous n'employions pas de principes de volition ou de raison pratique qui nous permettent de déterminer nos raisons d'agir.

$4 v)$ Nous ne pourrions pas employer de principes de volition ou de raison pratique, et a fortiori déterminer nos raisons d'agir, si nous n'avions pas une identité pratique de caractère normatif (une identité normative).

$5 \mathrm{v})$ Nous ne pourrions pas avoir une identité normative si, ayant réfléchi sur notre propre identité, nous n'attribuions pas de valeur à notre humanité (si nous ne nous « endossions pas nous mêmes de façon réfléchie » comme des êtres ayant de la valeur - si nous ne "trouvions " pas que notre propre humanité a de la valeur).

6v) Nous ne pourrions pas attribuer de valeur à notre propre humanité si nous n'attribuions pas de valeur à tous les êtres humains (si nous ne " trouvions » pas que tous les êtres humains ont de la valeur).

$7 v$ ) Nous devons trouver que (tous) les êtres humains ont de la valeur.

$8 v$ ) Nous trouvons que les êtres humains ont de la valeur.

9v) Les êtres humains ont de la valeur.

\section{Conclusion :}

10v) L'obligation morale - la force des normes morales — dérive de la nature de notre agir rationnel qui nous impose l'attribution de la valeur à nousmêmes ${ }^{17}$.

L'évaluation de cet argument assez complexe pose plusieurs problèmes qu'on ne peut pas traiter ici de manière adéquate. Nous allons nous limiter à une discussion critique des aspects du contenu des prémisses et de la forme des transitions logiques qui concernent son caractère transcendantal. Nous voulons également prendre en considération des remarques, des objections,

17. Voir Korsgaard, The Sources of Normativity, passim. La reconstruction proposée se base en partie sur le résumé de l'argument fait par Cohen G.A. ibid., p.185 et sur la discussion de Allan Gibbard (cf. Gibbard, Allan, "Morality as Consistency in Living : Korsgaard's Kantian Lectures ", Ethics 110, 1999, p. 140-164). Pour la formulation de certaines prémisses (2c-4c), on devrait compléter le raisonnement développé dans The Sources of Normativity par la lecture de Kant proposée par Korsgaard dans ses autres ouvrages, notamment dans Creating the Kingdom of Ends. Cf. aussi Korsgaard, Christine, "Motivation, Metaphysics and the Value of the Self : A Reply to Ginsborg, Guyer and Schneewind ", (dans Symposium on Christine Korsgaard's Creating the Kingdom of Ends) Ethics, 109, 1998, p. 49-66, (50-51). Voir aussi Korsgaard, The Sources of Normativity, p. 243 sq. J'ai décidé d'adopter une traduction littérale des expressions employées par Korsgaard ( « reflective endorsement », "we find our own humanity valuable ») afin d'essayer de fournir une description de ses positions aussi exacte que possible. 
des parallèles et des conceptions alternatives qui pourraient mettre en évidence des préoccupations auxquelles nous avons déjà fait allusion à l'égard du schéma conatif proposé par Moore. Comment devrait-on concevoir l'extension du prénom qui se réfère aux sujets humains constituant des agents rationnels ? Quelle est la nature et la portée des modalités invoquées afin de montrer les liens nécessaires entre les concepts que l'on propose d'élucider ? Quel est le statut métaphysique des faits décrits dans les prémisses adoptées de manière explicite ou tacite, et des faits supposés établis dans la conclusion ? Jusqu'à quel point pourrait-on assurer la validité de la dérivation accomplie ? Quelle est la force de la conclusion et dans quelle mesure dépend-elle du caractère transcendantal du raisonnement employé ?

\subsubsection{Une fondation subjective et volontariste de l'objectivité morale?}

On doit commencer par noter que le "nous " dont il est question dans l'argument $(\mathrm{V})$ - mais aussi dans ses formes plus courtes $(\mathrm{A})$ et $(\mathrm{B})$ - désigne tout être humain pourvu de raison pratique. En tout cas, il semble qu'on ne rencontrerait aucune difficulté dans l'attribution commune de la valeur à notre humanité, dans la mesure où cette humanité est partagée par tous et où on ne risque pas de se borner à une référence exclusive à soi, de se trouver dans une situation où chacun privilégie sa propre humanité, conçue séparément et indépendamment de celle des autres ${ }^{18}$. Néanmoins, il n'est pas évident que Korsgaard puisse établir le lien transcendantal entre la conception de notre identité normative et l'attribution de la valeur à notre humanité commune sans un argument supplémentaire qui montre la nécessité de la transition d'un idéal souvent de caractère personnel, ou même relatif par rapport à une communauté restreinte, aux exigences de la raison pratique qui nous impose des valeurs et des normes universelles qu'il serait juste de reconnaître comme morales. Autrement dit, il faut voir comment (6v) découle de $(5 \mathrm{v})$, comment on procède pour déduire la loi morale à partir de notre réflexion sur nos identités plus ou moins contingentes. On devra bien sûr se référer à l'emploi du théorème kantien de l'universalisabilité nécessaire de nos maximes, qui est codifié dans la première formulation de l'impératif catégorique, comme la première étape de l'institution du "Royaume des Fins ». Le problème est que l'utilisation de ce théorème ne peut pas garantir par elle-même la fondation de ce Royaume et l'intention de s'y installer.

C'est sur ce point que l'on est obligé de faire face à des difficultés bien connues depuis les premières tentatives d'interprétation et d'évaluation des positions kantiennes et de leurs analogues contemporains. La lecture de Kant

18. Voir l'observation de Moore sur ce point ; cf. Moore, "Conative Transcendental Arguments... ", p. 291. Pourtant Moore ne prend pas en considération la question difficile des relations entre nos conceptions personnelles et peut-être divergentes de "notre identité normative » et cette humanité commune. Voir la discussion qui suit. 
par Korsgaard essaie de minimiser, sinon d'éliminer, tout appel à des notions métaphysiques qui pourraient menacer l'autonomie de la raison pratique. Elle s'oppose à tout intuitionnisme rationaliste qui rendrait possible la défense de quelque forme de réalisme «substantiel» en éthique, quoiqu'elle accepte ce qu'elle appelle le " réalisme procédural », c'est-à-dire la position selon laquelle il y a des réponses aux questions morales parce qu'il y a des procédures correctes pour les atteindre. Elle rejette d'emblée toutes les versions de réalisme substantiel qui impliqueraient l'existence des faits moraux indépendants ou des vérités que nos procédures nous permettraient de détecter ${ }^{19}$. En effet, on doit se demander si son traitement transcendantal de la «question normative " aboutit à des conclusions solides, précisément dans la mesure où elle ne veut pas aller au-delà des sources subjectives et des procédures de construction dont, suivant son analyse, dépend entièrement l'attribution de la valeur à notre humanité.

Selon Korsgaard, il suffit de renvoyer à l'autorité de nos pouvoirs de réflexion mis en œuvre par notre volonté pour accomplir la dérivation de l'impératif catégorique et de l'extension de cet impératif à la loi morale qui engage tout être rationnel. Ici, il importe de faire remarquer qu'elle nous appelle à distinguer clairement entre l'impératif catégorique qui fournit la structure des règles régissant la volonté libre de chaque personne douée de raison pratique et la loi morale proprement dite, qui régit effectivement le domaine entier de la communauté de telles personnes. Le besoin d'éliminer toute contradiction éventuelle dans l'opération de notre volonté dirigeant l'action indique seulement le critère qui, selon Kant, nous permet de décider quelles maximes pourraient être acceptées comme lois du «Royaume des Fins ». On devrait peut-être accepter cette épreuve de l'impératif catégorique, afin de spécifier les conditions nécessaires, quoique non suffisantes, pour la détermination du contenu des lois en question, tout en reconnaissant la possibilité d'avoir recours à des ressources et à des procédures alternatives qui achèveraient la tâche de la législation morale recherchée. Cependant, on serait

19. L'exemple le plus récent d'un tel réalisme, élaboré à partir des prémisses rationalistes, qui constitue l'objet de son attaque, est le réalisme normatif de Thomas Nagel. Le débat avec Nagel se poursuit, dans le contexte des réactions de Korsgaard aux critiques de son argumentation développée dans The Sources of Normativity. Voir Korsgaard, The Sources of Normativity, $\$ 1.4 .1-1.4 .9$, p. 28-47, p. 35-37 sur la distinction entre " réalisme procédural » et « réalisme substantiel ». Pour la discussion des positions de Nagel, voir pp. 40-44 et 242-251 ( "Reply to Nagel »). Voir aussi la critique de Nagel, " Universality and the Reflective Self », dans Korsgaard, The Sources of Normativity, p. 200-209. Cf. Nagel, Thomas, The View from Nowhere, Oxford, Oxford University Press, 1986, p. 139-140 (Le point de vue de nulle part, trad. française par Sonia Kronlund, Combas, Édition de Éclat, 1993) et l'expression modifiée de son approche dans son ouvrage le plus récent, The Last Word, Oxford, Oxford University Press, 1997. Pour une autre discussion des caractéristiques du réalisme normatif de Nagel et de l'ambivalence de certaines de ses positions, voir Virvidakis, La robustesse du bien, p.236-245 et "Stratégies de modération du réalisme moral », dans Ogien, Ruwen, dir., Le réalisme moral, p. 420-456. Voir aussi l'analyse qui suit $(3.1 .2,3.2)$. 
encore en quête d'un autre argument qui établirait la raison pour laquelle nous serions obligés de devenir citoyens de ce Royaume, c'est-à-dire de nous soumettre à l'autorité de la loi morale ${ }^{20}$. C'est justement afin de construire un tel argument que Korsgaard introduit sa conception de l'identité pratique, qui se présente sous la forme d'une identité normative et qui, suivant son raisonnement, est présupposée par toute action rationnelle. Malheureusement, bien que l'attribution de la valeur à cette identité semble effectivement fournir le fondement existentiel de l'agir rationnel, ce n'est pas du tout évident que la fonction de notre volonté en tant que source de toute valeur puisse supprimer, par elle-même, l'écart entre, d'une part, des raisons d'agir et des motifs basés sur des inclinations tout à fait égocentriques et, d'autre part, des raisons d'agir impartiales et objectives, capables de nous aider à juger et à rectifier ou à restreindre ces inclinations. C'est aux exigences des raisons d'agir proprement morales de caractère objectif que nous devons nous soumettre.

Ce problème, auquel on a déjà fait allusion plus haut en parlant de la difficulté d'assurer la validité de l'argument $(\mathrm{V})$ - en particulier concernant la transition de $(5 \mathrm{v})$ à $(6 \mathrm{v})$ - est souligné par Thomas Nagel dans sa critique de l'approche de Korsgaard ${ }^{21}$. Selon son analyse, elle semble s'inspirer d'une idée existentialiste assez éloignée de la pensée de Kant, dans la mesure où elle ne reconnaît aucune dimension indépendante de raisons d'agir en dehors de la nature de la volonté humaine qui constitue la seule source de la construction autonome de notre identité. Nagel croit qu'il existe de telles raisons d'agir nous permettant d'éviter le subjectivisme et le relativisme dans l'attribution de la valeur, non seulement à notre identité mais à l'humanité de tout être humain. Selon son approche, la réflexion sur l'éthique nous révèle des principes et des vérités qui «peuvent déterminer partiellement nos identités mais ne dérivent pas d'elles ${ }^{22}$. En plus, il insiste sur le fait que sa position réaliste à l'égard des raisons non subjectives ne l'engage pas forcément à adopter une métaphysique postulant l'existence de faits, d'entités ou de propriétés bizarres de caractère intrinsèquement normatif ${ }^{23}$.

Comme le suggère aussi G. A. Cohen dans sa discussion de l'argumentation de Korsgaard, ce qui manque à la dérivation de la valeur de l'humanité à partir de la réflexion sur la nature de notre identité pratique est l'appel kantien à un élément qui "transcende la nature humaine ", qui constitue la " souveraineté » de la raison, et qui s'impose à notre volonté « de l'extérieur ».

20. Cf. Korsgaard, The Sources of Normativity, \$3.2.4., p. 98-100.

21. Cf. Nagel, "Universality and the Reflective Self». Je trouve le qualificatif « existentialiste» (p. 203) assez juste. Il serait intéressant de comparer les suggestions de Korsgaard aux engagements et aux prétentions à l'universalisabilité de Sartre - qui sont, à vrai dire, présentés dans un contexte très différent de celui de la pensée kantienne. Cf. Sartre, J. P., L'existentialisme est un humanisme, Paris, Nagel, 1946.

22. Ibid., p. 206.

23. Ibid., p. 205. Cf. aussi, Nagel, The View from Nowhere, p. 140 et Virvidakis, La robustesse du bien, p. 236-245. 
Aucune lecture purement constructiviste de Kant ne pourrait rendre justice ni à l'évidence des prémisses quasi-axiomatiques qui servent de point de départ pour la construction ni à la rigidité de la structure de la raison pratique $^{24}$. On devrait peut-être avouer que le fait de la raison sur lequel se base et auquel doit se soumettre notre volonté rationnelle et libre ne consiste pas simplement en actes d'affirmation et en processus de construction de notre identité, comme semble le croire Korsgaard.

En effet, il ne suffit pas de constater que « la prétention à la généralité ou à l'universalité est essentielle à ce qui rend un acte, acte de la volonté " pour réussir dans la dérivation de l'engagement à la loi morale à partir de l'attribution de la valeur à notre identité pratique. Korsgaard elle-même ressent le besoin de développer des arguments supplémentaires afin d'établir ses conclusions concernant les règles constitutives de l'opération de la raison pratique. Cependant elle est persuadée que l'analyse de la conception de notre identité pratique, formée suivant la réflexion sur les sources de la valeur et l'endossement des résultats de cette réflexion par le sujet, révèle la nature, le fondement et la justification ultime de l'obligation morale 25 .

Le problème est que plusieurs formes et exemples particuliers d'identité normative paraissent tout à fait acceptables à ceux qui les endossent après avoir réfléchi, sans que leur attribution de la valeur à eux-mêmes soit accompagnée d'une attribution de la valeur semblable à l'humanité commune de tous les gens. Ainsi un général nationaliste serbe est-il capable d'endosser une notion assez claire de son identité nationale et culturelle, qui détermine les règles de son comportement mais qui entraine toutefois une éthique communautarienne et particulariste allant à l'encontre du respect d'autres êtres humains et de la loi morale telle que nous la concevons depuis Kant. Un nazi prêt à défendre la cohérence de ses actes de volonté et de ses actions cruelles pourrait même vouloir universaliser ses maximes, exprimant sa fierté à l'égard de son identité imaginaire politique et raciale, à laquelle il n'hésite pas à attribuer de la valeur ${ }^{26}$. Certainement, un philosophe kantien serait convaincu que

24. Cf. Cohen, G. A. «Reason, Humanity and the Moral Law », dans Korsgaard, The Sources of Normativity, p. 167-188, 170-177. Sur le besoin d'intégrer des éléments intuitionnistes, sinon platonistes, dans les modèles constructivistes pour les protéger du danger de se pencher vers le subjectivisme ou le relativisme, voir Virvidakis, La robustesse du bien, p. 249-251, et les références bibliographiques p. 250n. Voir aussi Virvidakis, "On Constructing and Discovering Moral Facts ", dans Funke, G., Akten des Siebenten Kant-Kongresses (Kurfürstliches Schloss zu Mainz, 1990), Bonn, Bouvier, 1991, p. 429-438.

25. Voir ses réponses aux objections dans The Sources of Normativity, p. 219-258, 232, 235 236 et son affirmation catégorique concernant le rôle central de l'endossement réfléchi dans l'institution de la morale : "So, the reflective endorsement test is not merely a way of justifying morality. It is morality itself » (ibid., p. 89). Cf. aussi Korsgaard, « Motivation, Metaphysics and the Value of the Self... », p. 66 ( « ...justification can come to an end only with a law you yourself will... »).

26. Pour d'autres exemples de ce genre, voir Cohen, « Reason, Humanity and the Moral Law ", p. 183-4, Gibbard, "Morality as Consistency in Living : Korsgaard's Kantian Lectures ", p. 154-155, 163. 
les maximes qui soutiennent les choix de telles personnes ne peuvent pas résister à l'épreuve de l'impératif catégorique et ne sont pas universalisables. Elles ne seraient pas sanctionnées par les principes de la raison pratique. Néanmoins, Korsgaard n'explique pas de manière convaincante comment l'appel à l'identité normative, essentiel à l'orientation de notre agir rationnel, s'avère efficace et adéquat pour justifier la soumission à l'autorité de la morale, sans une spécification préalable et substantielle de l'identité en question. La simple analyse de la prétention à l'universalité ne parvient pas à établir la conclusion requise et prête encore le flanc aux critiques classiques de la vacuité du formalisme ou du « logicisme moral ${ }^{27}$ souvent adressées à la philosophie morale kantienne. D’autre part, la référence métaphorique de Korsgaard au besoin d'intégration et de cohérence des " parties de l'âme ", qui renvoie à la conception de la santé de l'âme rationnelle d'après Platon ${ }^{28}$, trahit peut-être la dépendance tacite de sa notion d'identité à une perspective beaucoup plus objective et substantielle que l'activité autonome de détermination de soi et de l'attribution de la valeur à l'identité de soi-même.

\subsubsection{Le statut ontologique de la valeur attribuée à l'humanité}

Cependant, même si on parvient à établir la transition à la reconnaissance de l'autorité des demandes morales sans avoir recours à aucune version de réalisme autre que strictement " procédural », il ne faut pas sous-estimer les difficultés qui assaillent le reste de l'argument. Korsgaard présente l'attribution de la valeur à l'humanité de tous les êtres humains comme une condition nécessaire de l'attribution de la valeur à notre propre humanité (qui à son tour constitue la condition nécessaire de l'affirmation de notre identité normative, reconnue aussi dès le début comme une condition nécessaire de l'agir rationnel). Elle constate que cette attribution a lieu dans la mesure où nous reconnaissons ("trouvons») en effet que les êtres humains ont de la valeur $(8 \mathrm{v})$ et elle en infère ensuite l'existence actuelle de la valeur $(9 \mathrm{v})$. Elle défend cette inférence contre l'objection selon laquelle notre attribution ou reconnaissance subjective de la valeur ne nous permettrait pas de parler de détection ou de connaissance d'une qualité objective qui pourrait appartenir aux êtres humains en réalité. On ne devrait pas assimiler son argument au raisonnement problématique de Mill, qui visait à mettre en lumière ce qui est objectivement

27. On pourrait appeler « logicisme moral » la position selon laquelle la morale « fait simplement partie de la logique de ce que nous devons faire. »Cf Gibbard, "Morality as Consistency in Living : Korsgaard's Kantian Lectures », p. 143.

28. Korsgaard se réfère à la conception de l'âme de Platon dans sa réponse à Bernard Williams et tâche de limiter son appel à l'analogie à un sens plutôt formel, en admettant qu'elle ne peut pas pour le moment élucider les implications spécifiquement morales du principe du contrôle de soi et de l'harmonie de l'âme qu'elle trouve chez Platon. Cf. Williams, Bernard, "History, Morality and the Test of Reflection ", dans Korsgaard, The Sources of Normativity, p. 210-218, et ibid., p. 232-233. 
désirable en invoquant ce que tous les gens désirent, afin de «prouver » le principe d'utilité, puisque la dérivation transcendantale de la normativité ne se limite pas à la simple description d'un fait empirique, dont les implications normatives seraient acceptées seulement par les philosophes utilitaristes, mais se base sur l'inévitabilité de l'affirmation de notre identité normative en tant qu'êtres humains. En d'autres termes, il s'agit d'une déduction à partir de l'activité de notre réflexion sur la nature de l'agir rationnel, qui est censée expliquer et légitimer l'existence même de la valeur attribuée. Si on voulait prendre au sérieux le terme plutôt ambigu employé par Korsgaard, on pourrait dire que l'on «trouve » (« find») ce que l'on «place» soi-même. Il s'agit d'une sorte de projection et de découverte à la fois ${ }^{29}$. La réalité de la valeur en question est accessible seulement du point de vue de la première personne et de l'intérieur des formes de vie humaine, exactement comme la réalité phénoménologique des couleurs est accessible seulement du point de vue de la perception humaine et disparaît sous la perspective de l'analyse scientifique ${ }^{30}$. La question est de savoir si la position de Korsgaard, qui découle de son approche transcendantale, pourrait contourner les objections adressées à toute forme de réalisme ou d'objectivisme anthropocentrique qui se base, en dernière analyse, sur les critères épistémiques de la première personne.

\subsection{Les présupposés et les limites de l'argumentation transcendantale conative}

Nous pouvons maintenant élaborer nos remarques critiques sur le raisonnement de Korsgaard, afin, premièrement, de tâcher d'en dégager certaines conclusions en ce qui concerne les aspects caractéristiques de l'argumentation transcendantale conative mise en relief par Moore et, deuxièmement, d'éva-

29. Ici, on se rappelle la position apparemment paradoxale de David Wiggins, à michemin entre le subjectivisme et le réalisme, selon laquelle on crée et on découvre les valeurs à la fois. Voir Wiggins, David, "A Sensible Subjectivism ", dans Needs, Values, Truth, 2ème éd., Oxford, Blackwell, 1991, p. 185-214. Bien sûr, l'approche anthropocentrique de Wiggins est plus proche d'Aristote et de Hume et ne peut pas être assimilée au rationalisme kantien de Korsgaard. Cf. Virvidakis, La robustesse du bien, p. 144-209. Korsgaard pourrait s'accorder avec Michael Smith sur une conception de l'attribution de la valeur basée sur des croyances concernant ce qu'on désirerait si on possédait une rationalité "pleine » ou idéale. Cf. Smith, The Moral Problem, p. 147-177. Pourtant, sa stratégie transcendantale est très différente des méthodes de Smith. Voir la discussion qui suit.

30. Ibid., $\$ 3.4 .10$, p. 124-125. Sur le problème apparent du parallèle entre les étapes finales de l'argumentation transcendantale du genre conatif et la " preuve » de validité douteuse de Mill, voir aussi ibid., $\$ 2$ 24.1., p. 79-80, et les remarques de Moore, ci-dessus, note 8. La référence au parallèle avec les couleurs fait penser à toute une série d'arguments concernant l'analogie entre les propriétés morales et les qualités secondes comme les couleurs. Quoique la position de Korsgaard, se rapproche d'une forme de réalisme modéré concernant la normativité, elle est très différente des modèles de réalisme moral dispositionnel qui se basent sur l'analogie avec les couleurs, employée par Wiggins et John McDowell. Cf. Virvidakis, La robustesse $d u$ bien, p. 150-176. Voir aussi la note précédente. 
luer l'apport de cette forme d'argumentation aux tentatives d'explication et de justification de l'autorité des normes morales. Comme nous l'avons déjà suggéré au début de notre discussion, nous devons prendre en considération des approches de la raison pratique complémentaires ou alternatives.

Il est clair que, même si on accepte la validité des transitions logiques effectuées entre les diverses prémisses de l'argument développé par Korsgaard, on doit s'accorder sur le contenu et le statut épistémique et modal des prémisses. Comme dans tout argument transcendantal, la nécessité des conditions invoquées et des positions défendues n'est ni strictement logique ou conceptuelle ni simplement psychologique. Cette ambiguité de la modalité en question, qui de prime abord paraît comme un défaut dans l'articulation du raisonnement, pourrait en fait indiquer le caractère particulier de l'approche anthropocentrique adoptée permettant la justification de la normativité morale sans faire appel à des assertions dogmatiques invoquant nos intuitions réalistes.

Si la source ultime de la valeur de notre humanité commune et de la distinction entre ce qui est et ce qui n'est pas moralement juste est tout simplement le sujet de la réflexion qui procède à endosser sa propre rationalité, nous n'avons peut-être besoin d'aucune autre fondation naturelle ou surnaturelle de l'obligation de nous soumettre aux demandes de la loi morale. Nous « devons » réfléchir sur notre identité normative, en tant qu'agents rationnels, et nous " devons » lui attribuer de la valeur, dans la mesure où nous acceptons de continuer à mener notre existence contingente. C'est pourquoi la nécessité de la reconnaissance de la valeur que nous « trouvons » dans tous les êtres humains n'est pas conçue sub specie aeternitatis, mais plutôt sub specie humanitatis. Elle aussi, comme la loi morale, semble dériver de nos actes de volonté qui forgent nos identités de façon autonome, conformément à l'idée de la liberté qui constitue le fond de notre raison pratique.

Il n'y a de place pour aucune forme de réalisme ontologique substantiel si la stratégie argumentative transcendantale se base entièrement sur le postulat d'une activité de construction ou de projection de la valeur (constituant une forme d'expression rationnelle de nos états conatifs). En effet, Korsgaard rejette la distinction, acceptée par Nagel, entre des raisons d'agir (et des valeurs) relatives et des raisons d'agir (et des valeurs) neutres par rapport à l'agent, parce qu'elle est persuadée qu'il n'y a que des raisons que nous pouvons et devons partager, il n'y a que des valeurs morales que nous pouvons et devons créer et attribuer, en nous référant toujours à notre humanité commune ${ }^{31}$. D'après son analyse, les raisons normatives et les valeurs qui les

31.Voir Korsgaard, Christine, "The Reasons We Can Share : An Attack on the Distinction Between Agent-Relative and Agent-Neutral Values ", Social Philosophy and Policy, 10, 1993, p. 24-51, repris dans Korsgaard, Creating the Kingdom of Ends, chap. 12. Ici, il importe de noter le parallèle avec l'argument de Moore, selon lequel le raisonnement transcendantal conatif nous permet de nous débarrasser de l'opposition entre " raisons internes » et " raisons externes " déterminant la décision et l'action morale. Cf. ci-dessus, section 2 et note 11 . En ce 
étayent sont de caractère intersubjectif. Comme on l'a déjà vu, il ne pourrait pas y avoir de raisons d'agir purement objectives, conçues de l'extérieur de notre réflexion, du point de vue de la troisième personne. Pourtant, il n'y a pas non plus de raisons exclusivement personnelles ou égocentriques, comme il ne pourrait pas y avoir de langage privé ${ }^{32}$. En tout cas, la dimension intersubjective des raisons que nous sommes capables de partager devrait suffire pour étayer le réalisme "procédural » adopté par Korsgaard, selon lequel il existe des réponses correctes et incorrectes aux questions morales, réponses qui ne dépendent pas de nos goûts arbitraires ${ }^{33}$.

Néanmoins, la tentative ambitieuse d'une déduction transcendantale de l'autorité, sinon de la souveraineté, de la normativité morale qui fait appel aux conditions de possibilité de l'identité normative de tout agent rationnel ne peut pas réussir si elle se limite à une perspective entièrement constructiviste. Comme on l'a déjà noté, on ne peut pas établir l'extension de l'attribution de la valeur à l'humanité de tous les êtres humains par la simple réflexion sur la structure de la notion d'identité normative. En plus, la défense du caractère public des raisons, qui invoque l'analogie avec l'argument wittgensteinien mettant en cause la possibilité d'un langage privé, ne parvient pas à démontrer l'incohérence de toute forme d'égoïsme et de relativisme ${ }^{34}$.

Ici, il importe de remarquer que la prise de conscience de ces problèmes n'entraîne pas forcément l'abandon de tout argument transcendantal conatif visant à répondre à la " question normative ". Cependant, on est obligé, ou bien de modifier les prémisses cruciales qui postulent des conditions de possibilité de nos concepts et de notre agir rationnel, ou bien de modérer les prétentions à la légitimation des pouvoirs de la raison pratique ${ }^{35}$.

La première voie que l'on pourrait suivre serait donc de persister dans la recherche des conditions de possibilité ou des éléments constitutifs de l'agir et de l'opération de notre volonté, tout en reconnaissant que les relations a priori que l'on parviendrait à mettre en lumière ne dépendent pas de notre réflexion et de nos actes d'endossement. Une telle approche signifierait pourtant l'adoption de certains présupposés réalistes qui ne seraient pas faci-

qui concerne le pouvoir motivationnel des jugements moraux, Korsgaard adopte une forme d'internalisme (idéal), quoiqu'elle préfère éviter d'aborder le débat, parce que la " question normative » qui la préoccupe concerne la reconnaissance et la sanction ultime de l'autorité des normes morales, étant donné que nous admettons leur pouvoir de nous inciter à l'action sans l'intermédiaire d'un facteur motivationnel tout à fait externe ou indépendant de nos croyances morales. Cf. Korsgaard, The Sources of Normativity, p. 81-82, 82n.

32. Voir l'emploi de l'argument de Wittgenstein concernant l'incohérence de la notion d'une langue privée dans le contexte de la défense du caractère public et intersubjectif des raisons normatives. Ibid., $\mathbb{\$} 4.2 .3$, p. 81-82.

33. Ibid., $\mathbb{\$} 1.4 .4$, p. 34-35.

34. Cf. Nagel, " Universality and the Reflective Self », p. 208.

35. Pour une modération des prétentions anti-sceptiques des arguments transcendantaux visant à établir les relations entre nos concepts les plus fondamentaux, voir Strawson, P.F., Scepticism and Naturalism : Some Varieties, New York, Columbia University Press, 1985. 
les à justifier. Comme on le constate quand on étudie les ouvrages récents de Nagel, le prix à payer serait peut-être un certain degré de dogmatisme, un appel direct à l'autonomie de la pensée morale substantielle et une incitation au quiétisme métaéthique, une affirmation de l'autorité du sens commun et des intuitions qui jouent un rôle décisif dans notre raisonnement pratique et semblent peser plus que n'importe quel doute sceptique ${ }^{36}$. La solution alternative proposée impliquerait un relâchement des exigences transcendantales et entraînerait la dérivation de conclusions moins fortes. Ainsi pourrait-on établir des relations constitutives ou a priori entre les composantes de notre pensée éthique à partir de nos intuitions communes et des "platitudes » qui servent de base pour une première conception de la moralité. On serait pourtant prêt à admettre, comme le fait Michael Smith, que l'on devrait avoir recours à l'expérience et suivre le développement actuel et la mise à l'épreuve pratique des théories morales, afin de confirmer ou de falsifier nos énoncés concernant l'existence des raisons normatives, telles que nous les concevons du point de vue d'un agent doué de rationalité idéale ${ }^{37}$. On pourrait également soutenir que la conception et surtout l'application de tout principe contractualiste (comme ceux élaborés par John Rawls et Jürgen Habermas) présupposent une base morale qui renvoie à la notion kantienne du respect inconditionnel des personnes qui voudraient l'adopter ${ }^{38}$. Cependant, cette base morale est acceptée de façon plus ou moins contingente et pragmatique et n'a pas le caractère universel ou même nécessaire auquel on prétend souvent aspirer quand on entreprend la construction d'une théorie éthique.

36. Cf. Nagel, The Last Word, chap. 6. Voir aussi Dworkin, Ronald, "Objectivity and Truth : You'd Better Believe It ", Philosophy and Public Affairs, 25, 1996, p. 87-139.

37. Cf. Smith, Michael, The Moral Problem, Oxford, Blackwell, 1994, p. 182-202.

38. Voir Larmore, Charles, "The Moral Basis of Political Liberalism », The Journal of Philosophy, 96, 1999, p. 599-625. Larmore discute le principe libéral de légitimité de Rawls (présenté dans Political Liberalism, New York, Columbia, 1993) et le principe du discours D de Habermas (élaboré dans Faktizität und Geltung, Suhrkamp, Frankfurt, 1993) qui révèlent leur désir d'éviter tout engagement métaphysique ou moral substantiel. Il pourrait également se référer à la conception d'un principe de base contractuel, proposé par Scanlon. Cf Scanlon, Thomas, What We Owe to Each Other, Cambridge Mass., Harvard University Press, 1999, et pour une première critique, Pettit, Philip, "Two Construals of Scanlon's Contractialism ", The Journal of Philosophy, 97, 2000, p. 148-164. Toutes ces approches, qui visent à une légitimation des obligations morales par le biais de l'appel à une sorte d'accord hypothétique sur les normes que des êtres rationnels et raisonnables seraient enclins à accepter ou au moins à ne pas rejeter, pourvu qu'elles soient acceptées aussi par les autres, pourraient être considérées comme des descendantes de la famille de positions que Korsgaard appelle "volontarisme » et dont elle doute de la plausibilité en tant que réponse à la « question normative ». Cf. Korsgaard, The Sources of Normativity, p. 21-27. 


\section{Conclusions}

L'argumentation de Korsgaard nous fournit un exemple concret de l'application du modèle transcendantal conatif décrit par Moore. Ayant examiné sa structure et ses prémisses afin d'en identifier les avantages et les défauts, nous pouvons maintenant résumer nos conclusions modestes et provisoires, qui confirment en partie les observations initiales de Moore et permettent une première évaluation :

a) On peut essayer, en métaéthique, de construire des arguments valides qui sont conformes au modèle transcendantal, mais il est très difficile de s'accorder sur les prémisses de base et de trouver un point de départ assez solide pour résister de manière efficace aux attaques sceptiques. b) L'aspect conatif de tout argument de ce genre qui porte sur la nature de la raison pratique, aussi bien que la réflexivité des prémisses qui se réfèrent à la pratique des sujets dont il est question, se prêtent mieux à la défense des positions antiréalistes en éthique, quoique cet antiréalisme soit compatible avec quelques formes de réalisme modéré et anthropocentrique et aille de pair avec l'élaboration de certaines versions d'objectivisme ou d'intersubjectivisme. c) Il faut se méfier de tout argument qui prétend dégager des conclusions substantielles concernant le contenu et l'autorité des normes morales seulement à partir de la forme et de la structure a priori de la raison pratique et sans aucune référence aux données de l'expérience éthique.

Cependant, l'analyse de la réponse kantienne à la «question normative » proposée par Korsgaard ne suffit pas pour une appréciation juste du potentiel et des défauts des arguments transcendantaux en philosophie morale. On devrait procéder à une étude comparée d'une variété de tels arguments en philosophie morale, afin d'en établir la conformité au modèle conatif, et d'en repérer les prémisses communes et les points de convergence et de divergence, avant d'essayer de les évaluer. Si les doutes concernant leur validité et la plausibilité de leurs prémisses persistent, on serait d'abord obligé de réexaminer le modèle de Moore, adopté dès le début en tant que reconstruction du raisonnement transcendantal. En tout cas, il serait toujours possible d'employer des arguments transcendantaux conatifs qui ne conduisent pas à des solutions exclusives et définitives aux problèmes des théories normatives et métaéthiques, mais seulement afin d'élucider les relations de nos concepts centraux en philosophie pratique et d'explorer des interprétations complémentaires de nos principes moraux ${ }^{39}$.

39. Une version plus courte de cet article a été présentée au Deuxième Congrès International de la Société de Philosophie Analytique (SOPHA) à Brest (4-6 Septembre). Je remercie les participants au Congrès et plus particulièrement Steven Davis, Pascal Engel, Ulisses Moulines, Emmanuel Picavet, Claude Panaccio et Christine Tappolet de leurs questions et de leurs remarques critiques soulignant les faiblesses du modèle de l'argumentation transcendantale conative et de son emploi par Christine Korsgaard. Je remercie aussi les évaluateurs de la revue Philosophiques, aussi bien que Christine Tappolet de leurs suggestions pour l'amélioration de la structure et du style de mon exposé. 